\title{
Soft matter physics : Tools and mechanical models for living cellular aggregates
}

\section{Khalifat, Nada}

\section{6-11}

Khalifat , N, Beaune , G, Nagarajan , U , Winnik , F M \& Brochard-Wyart , F 2016 , ' Soft matter physics: Tools and mechanical models for living cellular aggregates ', Japanese journal of applied physics part 1-Regular papers short notes \& review papers, vol. 55 , no. 11,1102 A8 . https://doi.org/10.7567/JJAP.55.1102A8

http://hdl.handle.net/10138/308013

https://doi.org/10.7567/JJAP.55.1102A8

cc_by_nc_nd

acceptedVersion

Downloaded from Helda, University of Helsinki institutional repository.

This is an electronic reprint of the original article.

This reprint may differ from the original in pagination and typographic detail.

Please cite the original version. 


\title{
Soft matter physics: Tools and mechanical models for living cellular aggregates
}

\author{
Nada Khalifat ${ }^{\S \|}$, Grégory Beaune ${ }^{\dagger}$, Usharani Nagarajan ${ }^{\dagger}$, Françoise M. Winnik ${ }^{\dagger \ddagger}$, and \\ Françoise Brochard-Wyart ${ }^{*} ॥$ \\ $\S$ Institut Curie, PSL Research University, CNRS, UMR 168, F-75005 Paris, France \\ " Sorbonne Universitiés, UPMC Univ Paris 06, CNRS, UMR 168, F-75005 Paris, France \\ + WPI International Center for Materials Nanoarchitectonics (MANA), National Institute for \\ Materials Science (NIMS), 1-1 Namiki, Tsukuba, Ibaraki 305-0044, Japan \\ ₹ Department of Chemistry and Faculty of Pharmacy, University of Montreal, CP 6128 \\ Succursale Centre Ville, Montreal, QC H3C3J7, Canada
}

*francoise.brochard@curie.fr

\begin{abstract}
Tissues belong to the broad field of active matter, a novel class of non-equilibrium materials composed of many interacting units that individually consume energy and collectively generate motion or mechanical stresses. Active systems span an enormous range of length scales, from individual living cells, to tissues and organisms, to animal groups. We introduce the concept of biological tissues as examples of entangled active matter, where the units (cell) are bound by transient links. We focus here on the mechanical properties (surface tension, elasticity, and viscosity) of cells and tissues derived from measurements performed by the pipette aspiration technique. This approach has been very fruitful in unveiling striking analogies between the physics of inert soft matter (polymer, viscous pastes, and Silly Putty ${ }^{\circledR}$ ) and the behavior of biological tissues. The results obtained from such analogies suggest important implications in the fields of tissue engineering and development.
\end{abstract}

\section{Introduction}

Living cells are capable of sensing mechanical stimulations arising from both the external and physiological environments ${ }^{1}$. Depending on the magnitude, direction and distribution of these mechanical stimuli, cells can respond in a variety of ways ${ }^{2}$. The mechanical properties of individual cells can determine the structural integrity of whole tissues arising from the mechanical interactions between cells and the surrounding extracellular matrix. Mechanical loads exerted at the tissue level are transmitted to individual cells.Any deviation from cell mechanical properties can result in the breakdown of cell biological functions and possibly lead to several diseases considered major health risks such as cancer. For instance mechanical characterization of cells from the pleural fluids of patients revealed that the Young's modulus of cancer cells is considerably less than in benign cells ${ }^{3}$. And, in arthritis, there is an increase in stiffness and viscosity of the chondrocytes related to abnormal mechanical factors that change the cellular behavior of the cells causing joint degenerations 4. Thus, critical insights into diverse cellular processes and pathologies can be gained by understanding the biomechanics and mechanobiology of cell and tissue. The mechanical 
properties of cells are extracted from models that are based on the continuum approach. In this approach, the cell is assumed to have homogeneous material properties. Various models based on this continuum approach have been developed to characterize the mechanical properties of living cells when subjected to mechanical stimuli ${ }^{5}$.

Various experimental techniques have been developed to probe mechanically single cells and cell aggregates with forces and displacements. We present here several studies in which tools originally developed for studies in soft matter physics are used to characterize the mechanical properties of living matter, form single cells to tissues where cells are glued together by specific proteins. We focus on the pipette aspiration technique that yields the surface tension, elasticity, and viscosity of single cells ${ }^{6}$ and multicellular aggregates ${ }^{13}$. This straightforward method easily generates several mechanical properties of cells, for instance (i) by modeling the cell as a liquid drop, the micropipette aspiration experiments were analyzed using the Laplace law and the viscoelastic rheological models for the dynamics of aspiration and (ii) by observing the aspiration-driven penetration of cells into the micropipette and measuring the evolution over time of the cell penetration length, two types of cellular behavior were observed: cells, such as neutrophils 7,8 and erythrocytes, that have a liquid-like behavior ${ }^{9}$ and solid-like cells, such as chondrocytes ${ }^{10}$ and endothelial cells, that act as a continuum solid. ${ }^{11,12}$.

Studies of cellular aggregates by the micropipette aspiration technique have indicated that aggregates behave like "living" viscoelastic droplets ${ }^{13}$ : Just like silly putty pastes, they behave like gelly drops at short times and flow like a liquid at longer times. In contrast to viscous pastes, cell aggregates reinforce their mechanical properties as they are subjected to the aspiration pressure. This behaviour, which has never been observed with viscous pastes, reflects the mechanosensitive active response of the cells' acto-myosin cortex. Upon aspiration at a constant suction pressure, $\Delta P$, the aggregate penetrates into the micropipette if $\Delta P$ is larger than $\Delta P_{c}=2 \gamma\left(1 / R_{p}-1 / R\right)$, where $R_{p}$ and $R$ are the micropipette and aggregate radii, respectively, and $\gamma$ is the surface tension. From the dynamics of aspiration, one can measure the surface tension, the elastic modulus, the viscosity, and the tissue relaxation time.

\section{The analogy of tissues with soft matter physics}

The analogy between tissues and fluids has improved considerably our understanding of the morphogenesis and movement of biological tissue. It was evoked for the first time by Malcolm Steinberg ${ }^{14}$ who observed that, similarly to a liquid droplet, a tissue of lower surface tension engulfs a tissue of higher surface tension $\gamma^{15}$. Since then, there are numerous examples demonstrating that analogies with soft matter physics bring valuable insights into the rheological properties of tissues. At first sight, cell packing in a tissue is very similar to the packing of bubbles in foam (Figure.1.Analogy 1). This analogy has been used successfully to describe the static properties of cell configuration in tissue development, but it failed when applied to the dynamics of tissues. Foams are solid, and flow only above a yield stress $\sigma_{y}$. In contrast, tissues are ultra-viscous liquids (Figure.1. Analogy 2) similar to 
polymer melts: below a relaxation time $\tau$ ( few hours) a tissue behaves like rubber with an elastic modulus $E$. Above $\tau$, it flows like a liquid of viscosity $\eta=E \tau$. The difference between foams and tissues is due to the noise produced by live cells. Thermal agitation is too weak to allow the reorganization of foams subjected to mechanical stresses. The energy barriers corresponding to the reorganization shown in (Figure.1) are much larger then the thermal agitation energy $k_{B} T$ and the system is frozen. Since cells are active and produce a large noise, tissues can flow, however. The difference between cell "living" droplets and liquid droplets is due to the life of the cells. Cells are sensitive to the surrounding environment; they feel the rigidity and the forces applied on them. Therefore, such living drops can be described as "active" viscoelastic pastes, able to react to forces applied on them by a reinforcement of their mechanical properties.

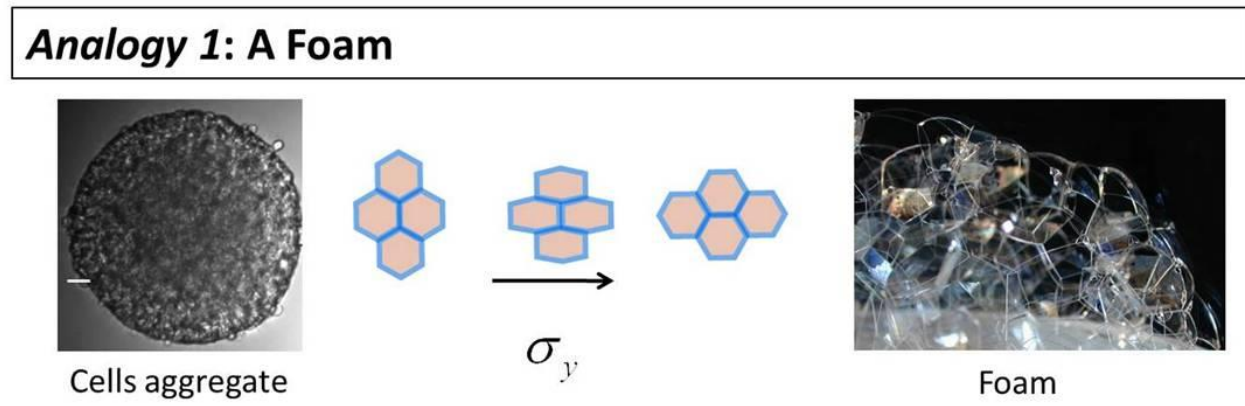

\section{Analogy 2: A Viscoelastic Paste}

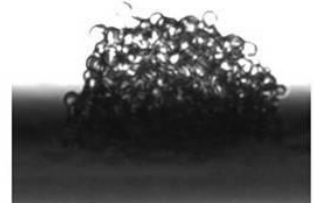

Spreading of a cells aggregate

$$
\frac{d \sigma}{d t}+\frac{\sigma}{\tau}=E \frac{d \varepsilon}{d t}
$$

Spreading of Silly Putty

Figure 1: Analogies of cellular aggregates. (A) Foam structure and (B) viscoelastic paste Silly Putty. Maxwell equation for the stress $\sigma$ versus the deformation $\varepsilon$, where $\tau$ is the tissue relaxation time, $E$ the elastic modulus, and $\eta=E \tau$ the viscosity.

Analogies between tissue mechanics and dynamical phenomena involving liquid interfaces, known as wetting phenomena, have been used to explain several ubiquitous tissue behaviors. Particularly striking is the analogy between tissue mechanics and liquid wetting found in tissue spreading. For instance, when two aggregates are brought into contact, they coalesce to form a single larger spheroid ${ }^{16}$, as observed in Fig 2 which depicts the spreading and fusion of two aggregates . 


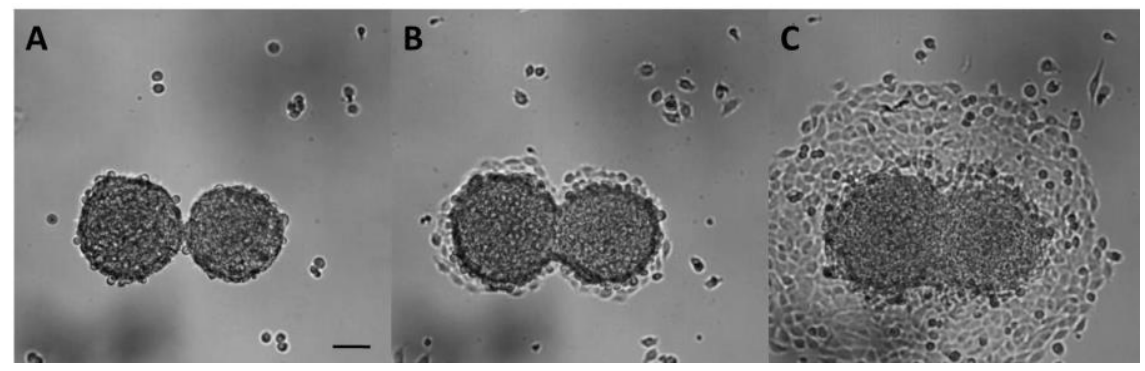

Figure 2: Fusion of two cellular aggregates. (A) Two aggregates in contact. (B) A neck connects the aggregates and (C) Spreading and fusion of the aggregates. The scale bar corresponding to $100 \mu \mathrm{m}$, courtesy S. Douezan.

When an aggregate is deposited on a substrate, two regimes can be observed, depending upon the sign of the spreading parameter $S . S=W_{c s^{-}} W_{c c}$ describes the competition between the cell substrate adhesion energy, $W_{c s}$, and the cell cell adhesion energy, $W_{c c}$. If $S<0$, cells prefer to stay in contact with other cells: the aggregate does not spread, which corresponds to the "partial wetting" situation. At equilibrium, the aggregate forms a spherical cap with a finite contact angle. Partial wetting is experimentally observed when a cell aggregate is placed on Pegylated poly(L-lysine) (PLL-PEG) coated surfaces. If $S>0$, the attraction to the substrate is dominant: the aggregate spreads with a precursor film consisting of a cell monolayer, a situation analogous to "complete wetting". The spreading parameter $S=W_{c s}$ $W_{c c}$ can be tuned by changing the cell surface adhesion $W_{c s}$, either chemically (PLL-PEG vs fibronectin) or physically, by changing the substrate rigidity ${ }^{17,18}$. The dynamics of the precursor film result from the balance between the gain of surface energy, and the viscous losses associated to the permeation of the cells from the (3D) aggregate into the (2D) film 18,19 . We have shown that the contribution due to the slippage of the monolayer that expands around the aggregates is negligible. On patterned substrates with adhesive strips separated by non-adhesive PLL-PEG bands (Fig. 13), we observed spreading of the monolayer on the stripe with a constant velocity $V^{*}\left(V^{*} \approx 7.910^{-9} \mathrm{~m} / \mathrm{s}\right.$ on glass coated with fibronectin), demonstrating that the permeation is the factor limiting the spreading ${ }^{18}$.

\section{The micropipette aspiration technique}

The tools used to examin cell mechanics can be classified in two sections summarized in (fig.3); (i) force application techniques, whereby a force is applied to a cell and the cell mechanical and/or biochemical response to this force is recorded And, (ii) force-sensing techniques, whereby cells are deposited on a soft substrate and the amplitude of the deformations is measured and used to evaluate the traction forces produced by cells during development, contraction, migration, and other commonly occurring cell processes. The disparity of experimental techniques and of applied loads that are used to explore mechanical response in the cell, elicit different mechanical responses in the cell. Thus, there are different mechanical models for living cells based (i) on the continuum approach ${ }^{5}$, and (ii) the Micro/Nanostructural Approach ${ }^{20}$. 

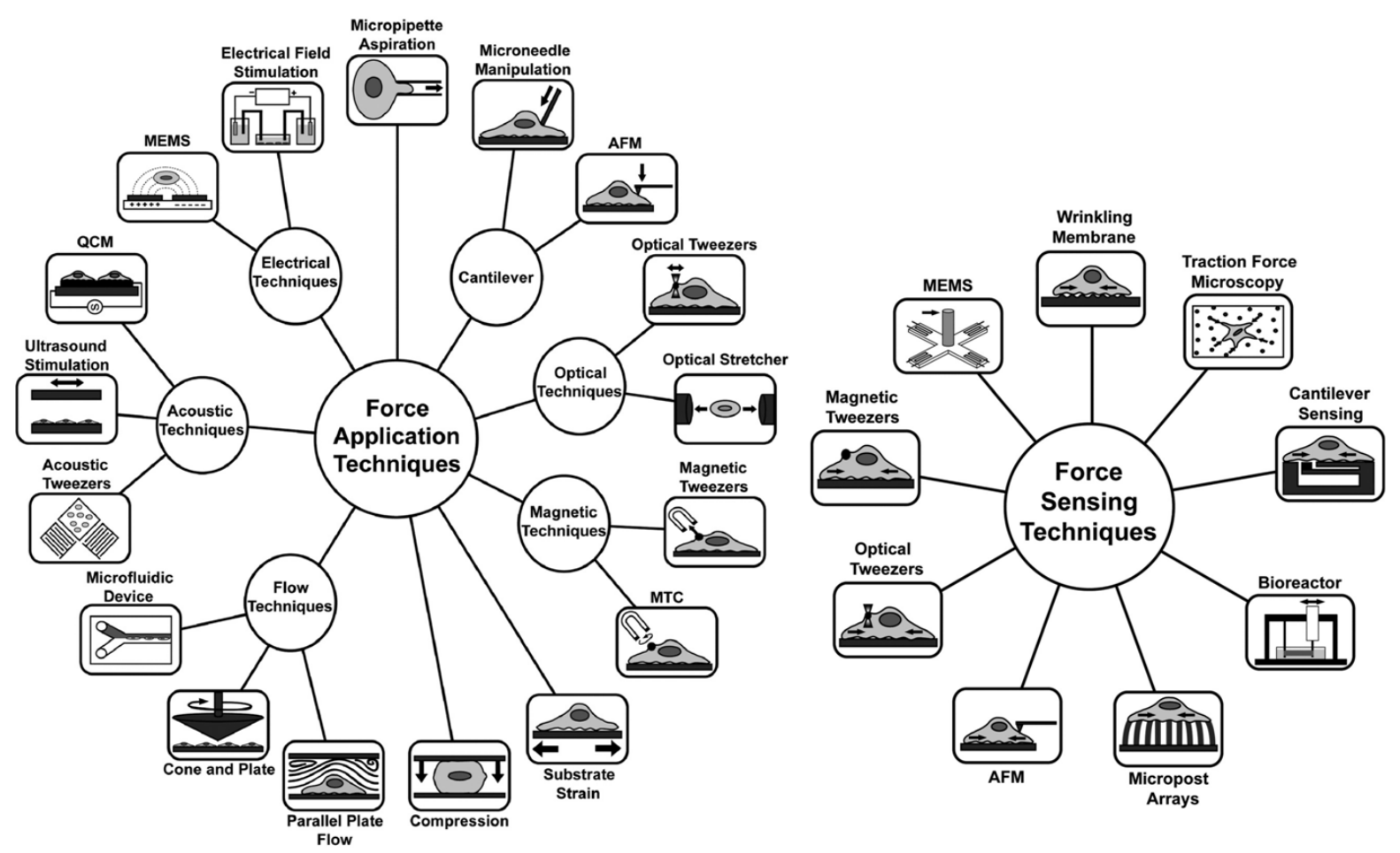

Figure 3: Experimental methods for measuring cell mechanics. Adapted from ${ }^{21}$.

The pipette aspiration technique is one of the force application techniques (1). In the following, we describe first the aspiration and extraction of simple oils, Then, we extend our study to active liquid drops.

\subsection{Liquid capillary aspiration and extraction}

A wetting liquid invades the capillary spontaneously and the penetration length increases as the square root of time in the case of a large drop or infinite reservoir ${ }^{22,23}$. This behavior is explained by the balance between the capillary forces and the viscous friction associated to the Poiseuille flow. For a small drop the penetration is enhanced by the Laplace pressure inside the drop ${ }^{24}$.

Non-wetting liquids enter in the capillary only under the action of an aspirating pressure. When such liquids are forced to penetrate the capillary, Capillary extraction is observed. This is the process by which a non-wetting liquid spontaneously leaves a capillary tube in order to minimize its surface energy ${ }^{25}$. For example, an oil slug initially placed at the end of the tube is unstable and comes out of the tube driven by surfaces forces (Fig.4). The liquid outside the tube forms a spherical cap of radius $R>R_{p}$, connected to a slug of length $\mathrm{L}$ ended by a hemisphere of radius $R_{p}$. Such a shape is not an equilibrium shape because of the difference of curvature between its ends. A Laplace pressure difference $\Delta P=$ $2 \gamma\left(1 / R_{p}-1 / R\right)$ is set between the drop extremities, which induces a flow from the small to the large cap. The driving force arising from the Laplace pressure difference can be 
written as: $F(x)=2 \pi \gamma R_{p}\left(1-\frac{R_{p}}{R(x)}\right)$. The motion stops when the drop is fully extracted at the exit of the tube. The origin of the motion can be easily understood: once a meniscus is out of the tube, any further displacement of the slug toward this meniscus reduces the surface energy of the system until the shape of minimum surface, i.e., a sphere, is attained. It has been shown that a drop in completely non-wetting state and placed at the end of a capillary tube leaves the latter spontaneously under the action of surface tension ${ }^{25}$. Unlike the capillary penetration of a wetting liquid, the extraction follows very different dynamics: its speed increases slowly from zero to a constant value. The primary cause of this difference is that the driving force is not constant during the experiment: it is close to zero at the beginning of the movement and grows slowly to a constant value as the drop gets out of the tube. The friction is different as well: the presence of a lubricating film between the drop and the capillary creates a plug flow in the droplet, unlike the Poiseuille flow in the capillary rise. Thus the dissipation at the exit of the tube becomes the main source of resistance if the oil is viscous.

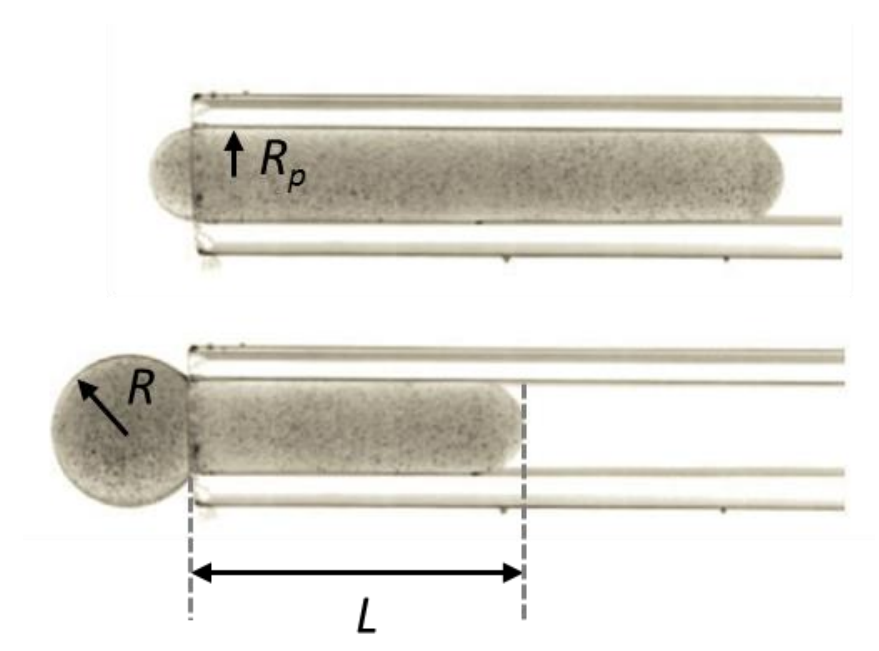

Figure 4: Extraction of a non-wetting liquid. Capillary extraction of non-wetting oil drop placed in a glass tube immersed in mixture of water ethanol solution of the same density with $L$ the length of the slug in the capillary and $R$ the radius of the spherical cap. Image time interval is $7.5 \mathrm{~s}$. Adapted from ${ }^{25}$.

\subsection{Micropipette aspiration of single living cells}

Micropipette aspiration is a simple and versatile tool to study the mechanical behavior of different cell types subjected to forces ranging from $10 \mathrm{pN}$ to $10^{4} \mathrm{nN}$. In a typical experiment, a capillary glass tube several microns wide at the tip is brought into contact with a cell. Then, a known suction pressure is applied within the micropipette, which is connected by some tubing to a water-filled reservoir of controllable height. A suction pressure is created within the micropipette by decreasing the height of the water surface in the reservoir relative to the height of the fluid surface in the cells observation dish. (Fig.5). Once the cell is drawn into the micropipette, several important mechanical characteristics of the 
cell can be obtained by measuring the length of the protrusion as function of time (Fig.4). If $\Delta P>\Delta P_{c}=2 \gamma\left(1 / R_{p}-1 / R\right)$, either the cell penetrates into the pipette as a viscoelastic liquid drop, or else the length reaches an equilibrium value, indicating that the cell is a viscoelastic solid (Fig.6). Solid-like behavior was observed for chondrocytes ${ }^{1,0}$ whereas neutrophils where shown to exhibit a liquid behavior ${ }^{7}$. When $\Delta P=0$, the cell exits the pipette like an oil droplet in capillary extraction (Fig.4). Cell mechanical models used to model the aspiration and the retraction are shown in fig 6 .

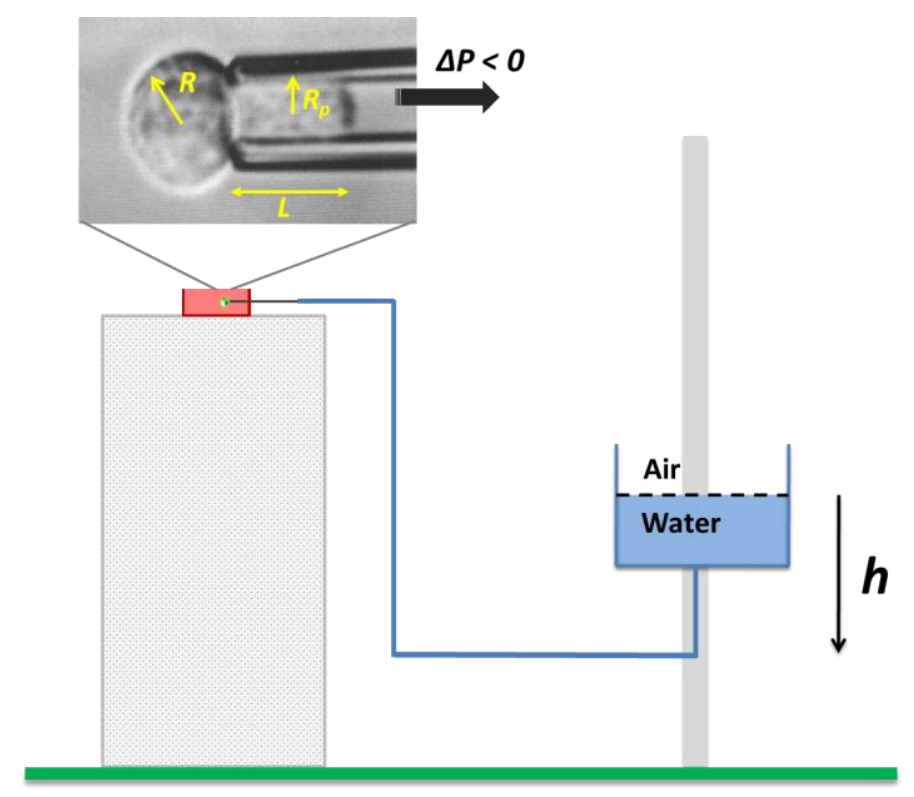

Figure 5: Sketch of the experimental setup of the micropipette aspiration technique and an image of aspiration $(\Delta P<0)$ of blood granulocytes adapted from ${ }^{26}$.

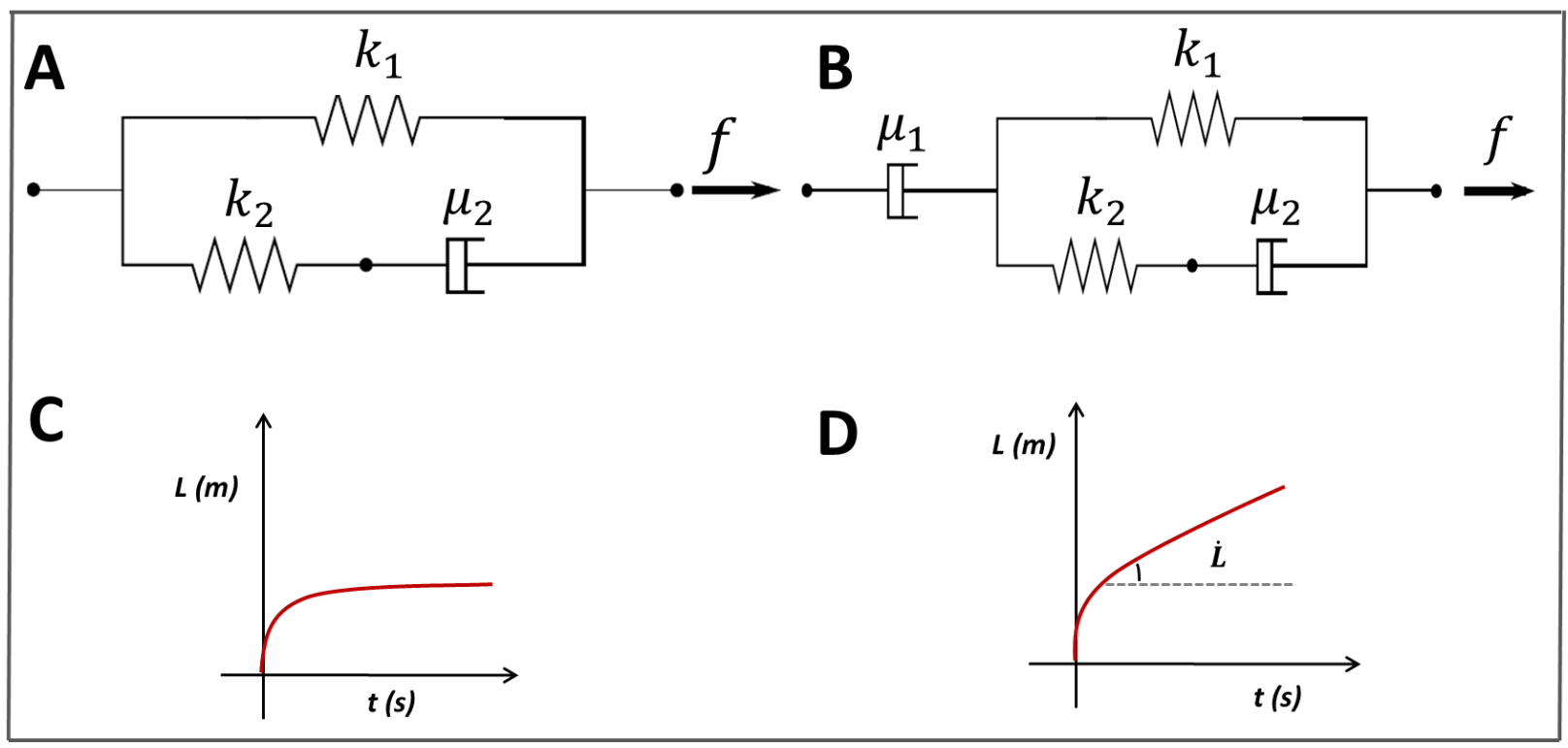

Figure 6: Mechanical model to characterize cell's behavior. The cell is modeled as homogeneous viscoelastic solid: Maxwell model (A), and as a viscoelastic liquid (B): modified Maxwell model, where $k_{1}$ is the spring constant related to the elasticity of the cell, $k_{2}$ 
accounts for the initial jump in $L(t), \mu_{2}$ is the local friction coefficient due to the raising time of the elastic deformation, and $\mu_{1}$ is the viscous dissipation of the flowing cell. Creep curves showing a fast elastic deformation: (C) before reaching equilibrium, and (D) followed by a viscous flow.

\subsection{Micropipette aspiration of cellular aggregates}

The mechanical properties of tissues are usually obtained via the parallel plate compression method, which involves the compression at constant deformation of a cellular aggregate placed between two non-adhering parallel plates ${ }^{27,28,29}$. By measuring the evolution of the compression force as a function of time, it is possible to obtain the viscoelastic properties, namely the elastic modulus $E$ and the viscosity $\eta$, of an aggregate. The surface tension $\gamma$ is obtained from the shape assumed by the aggregate at equilibrium, which can be challenging to determine experimentally. ${ }^{30}$ Another drawback of the technique is that it cannot be applied in vivo. Aggregate centrifugation is another experimental technique to quantify the mechanical properties of model tissues. In this case, the tissue surface tension $\gamma$ is obtained from the deformed shape of an aggregate under centrifugation, 31,32,33,34 Calculation of the fracture energy in partially fused aggregates has been used ${ }^{35}$ as well as the micropipette aspiration which is described next ${ }^{13,36,37,38}$. An overview of these techniques is given in (Fig.6).

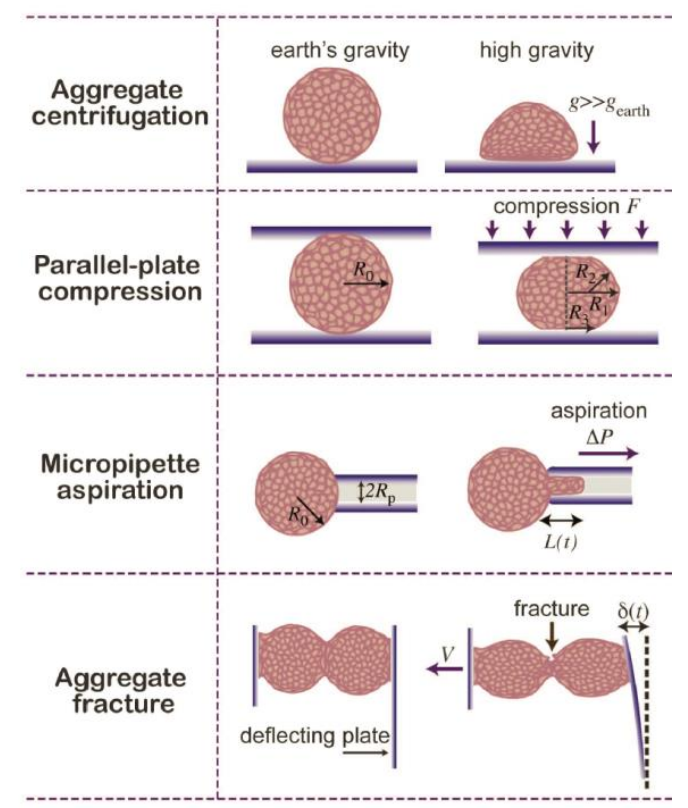

Figure 6: Experimental techniques used to characterize the mechanical properties of cell aggregates. Adapted from ${ }^{39}$.

In the micropipette aspiration ${ }^{13}$, the aggregate is sucked at constant pressure into a pipette of diameter smaller than the aggregate radius or diameter ??. The length of the aspirated tongue $L(t)$, is monitored as a function of time (Fig.7). The aggregate penetrates in the pipette if $\Delta P$ is larger than $\Delta P_{c}=2 \gamma(1 / R p-1 / R)$, where $R_{p}$ and $R$ are the micropipette and 
aggregate radii respectively, which allows one to determine the surface tension $\gamma$ of the aggegate. The elastic modulus $E$ and the viscosity $\eta$ are derived from the short term and long term aspiration dynamics, The aspiration and retraction curves are fitted by the viscoelastic Maxwell model shown in figure 4.B. From the data plotted in (fig. 7B) one obtains $\eta \approx 1.9 \times 10^{5}$ Pa.s and $E \approx 700 \mathrm{~Pa}$. However, cellular aggregates do not behave as simple viscoelastic drops, as a result of cell activity as discussed above. First, the surface tension $\gamma$ increases from $5 \mathrm{mN} / \mathrm{m}$ to $20 \mathrm{mN} / \mathrm{m}$ with $\Delta P$, showing that cells stretched in the capillary react by a reinforcement of the cortex which regulates the tissue surface tension. Second, in a narrow range of pressure $\Delta P$, pulsed contraction or "shivering" of the aggregate occurs, a response similar to the observed cell pulsation attributed to forces exerted between cells in developing tissue ${ }^{40}$.
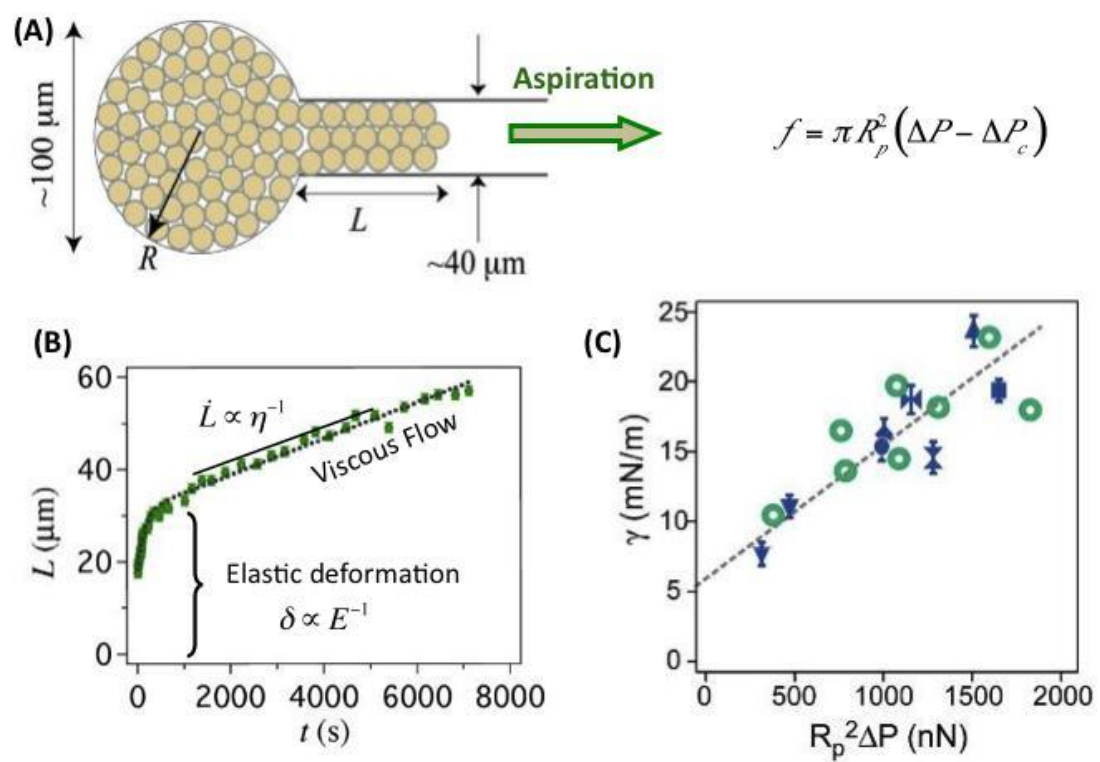

Figure 7: Cell aggregate aspiration. (A) Illustration of micropipette aspiration of spherical cellular aggregate. $\Delta P_{c}=2 \quad\left(1 / R_{p}-1 / R\right)$ is the threshold aspiration pressure. (B) Aspiration cycle for an aggregate $\Delta P=1180 \mathrm{~Pa}$, with $R=175 \mu \mathrm{m}$, and $R_{p}=35 \mu \mathrm{m}$ and (C) surface tension $\gamma$ $(\mathrm{mN})$ as function of applied force $R_{p}{ }^{2} \Delta P$. Adapted from ${ }^{13}$.

Complete aspiration of aggregates inside a pipette can be used to apply high pressures to cancerous tissues in order to investigate the validity of the homeostatic pressure model, which predicts that metastatic cells can only grow if the internal pressure of the aggregate is below a critical "homeostatic pressure". Combined with confocal microscopy, tissue relaxation under stress can be studied on the microscopic level by probing the cellular rearrangements inside an aspirated aggregate. Compared to more conventional methods, the micropipette aspiration technique is easy to set up and can be applied to the in vivo examination of biological systems, such as living tissue or drug treated tumors, and to other complex fluids, such as viscous pastes and foams. 


\section{Conclusion}

Analogies with soft matter, such as foams and pastes, have been applied to biological tissues to characterize their fundamental mechanical properties, such as surface tension, viscosity and elasticity. The framework of soft matter has been successful in explaining a number of dynamical tissue behaviors observed in physiology and development, such as cell sorting, tissue spreading, or the escape of individual cells from a tumor. However, living tissues exhibit active responses, such as rigidity sensing or cell pulsation, that are absent in inert soft materials. Future experiments that allow discrimination between different constitutive models should be designed, such as investigations of the frequency response of tissues to forces.

\section{Acknowledgements}

1. Bao, G. \& Suresh, S. Cell and molecular mechanics of biological materials. Nature Materials 2, 715-725 (2003).

2. Janmey, P. A. \& McCulloch, C. A. Cell mechanics: integrating cell responses to mechanical stimuli. Annual review of biomedical engineering 9, 1-34 (2007).

3. Cross, S. E., Jin, Y.-S., Rao, J. \& Gimzewski, J. K. Nanomechanical analysis of cells from cancer patients. Nature nanotechnology 2, 780-3 (2007).

4. Trickey, W. R., Lee, G. M. \& Guilak, F. Viscoelastic properties of chondrocytes from normal and osteoarthritic human cartilage. Journal of orthopaedic research : official publication of the Orthopaedic Research Society 18, 891-8 (2000).

5. Lim, C. T., Zhou, E. H. \& Quek, S. T. Mechanical models for living cells--a review. Journal of biomechanics 39, 195-216 (2006).

6. Hochmuth, R. M. Micropipette aspiration of living cells. Journal of Biomechanics 33, 15-22 (2000).

7. Evans, E. \& Yeung, A. Apparent viscosity and cortical tension of blood granulocytes determined by micropipet aspiration. Biophysical journal 56, 151-60 (1989).

8. Needham, D. \& Hochmuth, R. M. Rapid Flow of Passive Neutrophils Into a $4 \mu \mathrm{m}$ Pipet and Measurement of Cytoplasmic Viscosity. Journal of Biomechanical Engineering 112, 269 (1990).

9. Hochmuth, R. M. Measuring the Mechanical Properties of Individual Human Blood Cells. Journal of Biomechanical Engineering 115, 515 (1993).

10. Jones, W. R. et al. Alterations in the Young's modulus and volumetric properties of chondrocytes isolated from normal and osteoarthritic human cartilage. Journal of Biomechanics 32, 119-127 (1999). 
11. Theret, D. P. Application of the Micropipette Technique to the Measurement of Cultured Porcine Aortic Endothelial Cell Viscoelastic Properties. Journal of Biomechanical Engineering 112, 263 (2008).

12. Sato, M., Levesque, M. J. \& Nerem, R. M. Micropipette aspiration of cultured bovine aortic endothelial cells exposed to shear stress. Arteriosclerosis, Thrombosis, and Vascular Biology 7, 276-286 (1987).

13. Guevorkian, K., Colbert, M.-J., Durth, M., Dufour, S. \& Brochard-Wyart, F. Aspiration of biological viscoelastic drops. Physical review letters 104, 218101 (2010).

14. Steinberg, M. S. Reconstruction of Tissues by Dissociated Cells. Science 141, 401-408 (1963).

15. Foty, R. A. \& Steinberg, M. S. Cadherin-mediated cell-cell adhesion and tissue segregation in relation to malignancy. The International journal of developmental biology 48, 397-409 (2004).

16. Douezan, S. \& Brochard-Wyart, F. Dewetting of cellular monolayers. The European physical journal. E, Soft matter 35, 34 (2012).

17. Douezan, S. et al. Spreading dynamics and wetting transition of cellular aggregates. Proceedings of the National Academy of Sciences of the United States of America 108, 7315-20 (2011).

18. Beaune, G. et al. How cells flow in the spreading of cellular aggregates. Proceedings of the National Academy of Sciences of the United States of America 111, 8055-60 (2014).

19. Douezan, S. \& Brochard-Wyart, F. Spreading dynamics of cellular aggregates confined to adhesive bands. The European physical journal. E, Soft matter 35, 116 (2012).

20. Stamenović, D. \& Ingber, D. E. Models of cytoskeletal mechanics of adherent cells. Biomechanics and modeling in mechanobiology 1, 95-108 (2002).

21. Rodriguez, M. L., McGarry, P. J. \& Sniadecki, N. J. Review on Cell Mechanics: Experimental and Modeling Approaches. Applied Mechanics Reviews 65, 060801 (2013).

22. Lucas, R. Über das zeitgesetz des kapillaren aufstiegs von flüssigkeiten. Kolloid Z 23, (1918).

23. Washburn, E. W. The Dynamics of Capillary Flow. Physical Review 17, 273-283 (1921).

24. Marmur, A. Penetration of a small drop into a capillary. Journal of Colloid and Interface Science 122, 209-219 (1988). 
25. Piroird, K., Clanet, C. \& Quéré, D. Capillary extraction. Langmuir : the ACS journal of surfaces and colloids 27, 9396-402 (2011).

26. Evans, E. \& Yeung, A. Apparent viscosity and cortical tension of blood granulocytes determined by micropipet aspiration. Biophysical journal 56, 151-60 (1989).

27. Forgacs, G., Foty, R. A., Shafrir, Y. \& Steinberg, M. S. Viscoelastic properties of living embryonic tissues: a quantitative study. Biophysical journal 74, 2227-34 (1998).

28. Foty, R., Forgacs, G., Pfleger, C. \& Steinberg, M. Liquid properties of embryonic tissues: Measurement of interfacial tensions. Physical review letters 72, 2298-2301 (1994).

29. Foty, R. A., Pfleger, C. M., Forgacs, G. \& Steinberg, M. S. Surface tensions of embryonic tissues predict their mutual envelopment behavior. Development (Cambridge, England) 122, 1611-20 (1996).

30. Mgharbel, A., Delanoë-Ayari, H. \& Rieu, J.-P. Measuring accurately liquid and tissue surface tension with a compression plate tensiometer. HFSP journal 3, 213-21 (2009).

31. Phillips, H. M. \& Steinberg, M. S. Embryonic tissues as elasticoviscous liquids. I. Rapid and slow shape changes in centrifuged cell aggregates. Journal of cell science $\mathbf{3 0 , 1 - 2 0}$ (1978).

32. Phillips, H. M. \& Steinberg, M. S. EQUILIBRIUM MEASUREMENTS OF EMBRYONIC CHICK CELL ADHESIVENESS, I. SHAPE EQUILIBRIUM IN CENTRIFUGAL FIELDS. Proceedings of the National Academy of Sciences 64, 121-127 (1969).

33. Ninomiya, H. \& Winklbauer, R. Epithelial coating controls mesenchymal shape change through tissue-positioning effects and reduction of surface-minimizing tension. Nature cell biology 10, 61-9 (2008).

34. Kalantarian, A. et al. Axisymmetric drop shape analysis for estimating the surface tension of cell aggregates by centrifugation. Biophysical journal 96, 1606-16 (2009).

35. Wiebe, C. \& Brodland, G. W. Tensile properties of embryonic epithelia measured using a novel instrument. Journal of biomechanics 38, 2087-94 (2005).

36. Butcher, J. T., McQuinn, T. C., Sedmera, D., Turner, D. \& Markwald, R. R. Transitions in early embryonic atrioventricular valvular function correspond with changes in cushion biomechanics that are predictable by tissue composition. Circulation research 100, 1503-11 (2007).

37. Von Dassow, M. \& Davidson, L. A. Natural variation in embryo mechanics: gastrulation in Xenopus laevis is highly robust to variation in tissue stiffness. Developmental dynamics : an official publication of the American Association of Anatomists 238, 2-18 (2009). 
38. Von Dassow, M., Strother, J. A. \& Davidson, L. A. Surprisingly simple mechanical behavior of a complex embryonic tissue. PloS one 5, e15359 (2010).

39. Gonzalez-Rodriguez, D., Guevorkian, K., Douezan, S. \& Brochard-Wyart, F. Soft matter models of developing tissues and tumors. Science (New York, N.Y.) 338, 910-7 (2012).

40. Solon, J., Kaya-Copur, A., Colombelli, J. \& Brunner, D. Pulsed forces timed by a ratchetlike mechanism drive directed tissue movement during dorsal closure. Cell 137, 133142 (2009). 